\title{
Multiplex volatile organic compound Raman sensing with nanophotonic slot waveguides functionalized with a mesoporous enrichment layer
}

\author{
Haolan ZhaO, ${ }^{1,2, *}$ (D) Bettina Baumgartner ${ }_{9}{ }^{3}$ Ali RaZa, ${ }^{1,2}$ (i) Andre Skirtach ${ }^{2,4}$ \\ Bernhard LENDL, ${ }^{3}$ AND RoEL BAETS ${ }^{1,2}$ (]) \\ ${ }^{1}$ Photonics Research Group, INTEC, Ghent University-IMEC, 9052 Ghent, Belgium \\ ${ }^{2}$ Center for Nano- and Biophotonics, Ghent University, 9052 Ghent, Belgium \\ ${ }^{3}$ Institute of Chemical Technologies and Analytics, Technische Universität Wien, 1060 Vienna, Austria \\ ${ }^{4}$ Department of Biotechnology, Ghent University, 9000 Ghent, Belgium \\ *Corresponding author: Haolan.zhao@ugent.be
}

We report, to the best of our knowledge, the first Raman sensor for broadband vapor-phase volatile organic compounds (VOCs) based on silicon nitride waveguides functionalized with a mesoporous silica top-cladding. The Raman response of low-concentration VOC gases is evanescently probed via a centimeter-long functionalized slot waveguide. Thanks to the four-orders-of-magnitude concentration factor offered by the mesoporous coating, the detection limit of acetone, ethanol, and isopropyl alcohol (IPA) is determined to be 594, 157, and 53 ppm, respectively, with 1 s measurement time in the current setup. Capable of multiplex gas sensing in a complex matrix through fast and reversible adsorption, the demonstration paves the way to on-site continuous VOC monitoring.

Volatile organic compounds (VOCs) are organic chemicals with a high vapor pressure at room temperature. VOCs are ubiquitous in indoor air as they are emitted by a multitude of sources, both anthropogenic and natural $[1,2]$. However, many VOCs can cause adverse health effects [3]. Several institutes, including the World Health Organization (WHO) and Environmental Protection Agency (EPA) in the U.S. have established guidelines on VOC concentrations [4]. Driven by the increasing awareness of environmental protection and personal safety, low-cost portable devices for continuous on-site VOC have been a major focus of sensor research in recent years. Indoor air is a complex matrix containing numerous trace-level VOCs among plenty of other chemical compounds. The challenge of a VOC sensor is to identify and quantify each VOC at low concentrations from a complex atmospheric ambient, which demands methods with high sensitivity and high specificity.

Gas chromatography with mass spectroscopy (GC-MS) is the most widely used technique for VOC detection. However,
GC-MS requires bulky instrumentation, and its physical dimension limits its applicability outside laboratories. Ionmobility spectrometry (IMS) is a promising technique for atmospheric sensing with the potential of miniaturization. However, IMS has a limited specificity, and it often encounters challenges in quantifying separate VOC components in complex samples [5,6]. Mid-infrared absorption spectroscopy has been implemented to VOC sensing $[7,8]$. Integrated midinfrared sources and detectors, however, are not widely available yet, hurdling the miniaturization of this method.

Raman spectroscopy is a versatile analytical tool that can provide information on molecular ro-vibrational transitions by measuring the frequency shift of laser photons upon inelastic scattering by analytes. In doing so it reveals molecular fingerprints, which have found broad applications in the biomedical and environmental areas [9]. The analytical prowess of Raman spectroscopy for gas sensing has been recognized [10,11]. However, the intrinsic weakness of Raman scattering demands highly sensitive equipment and optimal measurement conditions, which has challenged its application in low-concentration gas analysis. One approach to enhance the Raman signal is to tightly confine both the optical excitation and the analyte within waveguiding structures. This method dramatically enlarges the light-matter interaction volume, leading to much enhanced signals [12]. Raman spectroscopy on hollow-core fibers filled with analytes has allowed for sub-10 ppm trace-level gases and liquids [11]. However, gas samples need to be pressurized to be injected into the cores, making it less suitable for continuous VOC sensing.

Recently, the silicon nitride ( $\mathrm{SiN}$ ) waveguide platform has gained importance for waveguide-enhanced Raman spectroscopy (WERS). In this case, the evanescent tail of the guided mode is used to excite and collect the Raman signal. On the one hand, the SiN platform has become a mature platform, providing the required components for Raman spectroscopy, 
ranging from integrated lasers [13], spectrometers [14], and high-performance filters [15]. On the other hand, the relatively high refractive index of SiN contributes to a stronger signal, and the planar waveguide structures allow the convenient introduction of analytes. In the past years, SiN WERS has already been demonstrated for bulk liquid [16] and biological monolayers [17]. More recently, inspired by the technique of solid-phase extraction, chemical enrichment has been introduced to WERS. In this Letter, the SiN waveguide is coated with a sorbent layer, which significantly concentrates the analytes and leads to much improved signals. Multiple gases at the parts per billion level have also been probed, thanks to the hypersorbent polymer functionalized waveguides $[18,19]$. However, the sorbent layer in these reports is a specially designed polymer that binds selectively with organophosphates and other toxic substances. The polymer coating also exhibits a long enrichment and recovery time, precluding its application in VOC sensing.

In this contribution, we report for the first time, to the best of our knowledge, a proof-of-concept demonstration of multiplex VOC sensing with WERS using slot a $\mathrm{SiN}$ waveguide functionalized with a mesoporous silica top-cladding. We explore the degree by which the mesoporous coating enriches the VOC concentration and find a four-orders-of-magnitude concentration enhancement. The Raman spectra of trace levels of acetone, ethanol, and isopropyl alcohol (IPA) have been recorded.

The nanophotonic slot waveguide is chosen for Raman sensing for its high efficiency [20]. The waveguide is fabricated in the BioPIX pilot line in IMEC. A $300 \mathrm{~nm}$ thick $\mathrm{SiN}$ layer is deposited through plasma-enhanced chemical vapor deposition on a $3.3 \mu \mathrm{m}$ thick layer of silica. The slot waveguide is then patterned via deep-UV lithography and reactive ion etching. The waveguide has a nominal cross section of $w \times h=700 \times 300 \mathrm{~nm}$ with a slot of $150 \mathrm{~nm}$. The slot waveguide is first transitioned to a $700 \mathrm{~nm}$ wide strip waveguide and then tapered to a $3 \mu \mathrm{m}$ wide strip waveguide at both ends for coupling. The tapering section is top-covered with a $1 \mu \mathrm{m}$ thick silica, and the overall length of the sensing slot waveguide is $8 \mathrm{~mm}$ in length. A $100 \mathrm{~nm}$ ordered mesoporous silica coating with a 3D hexagonal structure is then spin-coated on top of the slot waveguide section with the same technique explained in detail in Refs. [21,22]. Subsequently, the internal surface of the coating is post-functionalized with hexamethyldisilazane in absolute acetone. The functionalization increases the hydrophobicity of the coating for two purposes: (1) the functionalization allows the enrichment of a broad range of VOCs, since most of them exhibit a hydrophobic character and (2) free $\mathrm{OH}$-groups of the silica film are saturated, which prevents degradation of mesoporous coating. The cross section of the final waveguide is shown in Fig. 1(b). The porosity of the coating is $50 \%$, and its refractive index is estimated to be 1.21 (at $785 \mathrm{~nm}$ ) through effective medium approximation [22,23]. The waveguide supports only the fundamental quasi-TE and the fundamental quasi-TM mode. The TE mode is preferred as it has a larger modal overlap (37\%) with the sorbent layer when pumped at $785 \mathrm{~nm}$. The propagation loss is found to be $5.2 \mathrm{~dB} / \mathrm{cm}$ in a cutback measurement in ambient air, and the coupling loss is $7 \mathrm{~dB} /$ facet.

We use a confocal Raman microscope (WITec Alpha 300 R) with a $785 \mathrm{~nm}$ pump laser (Toptica XTRA II) for our measurement, as shown in Fig. 1(a). The polarization of the pump beam is controlled by a $\lambda / 2$-plate to excite the fundamental TE

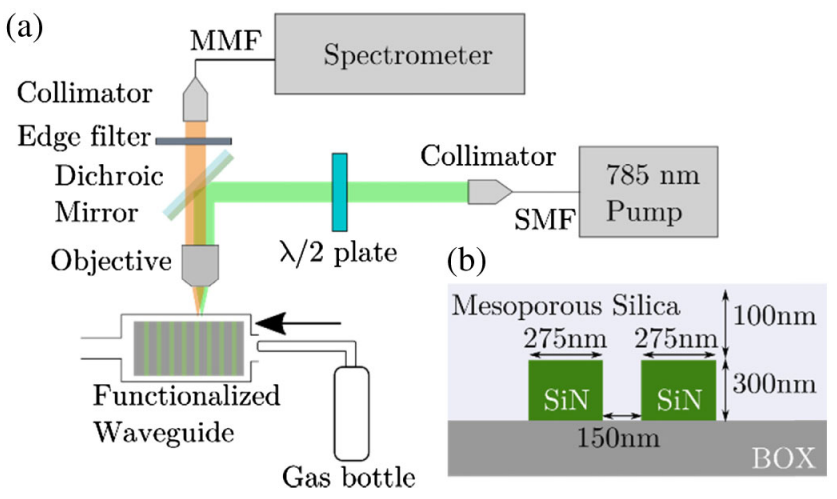

Fig. 1. (a) Setup for the gas measurement. Backward collection is used with the pump injected and the Stokes collected from the top facet of the waveguide. (b) Cross section of the slot waveguide for sensing.

mode of the waveguide. The pump power before the objective is measured to be $50 \mathrm{~mW}$. We use the backward-collection configuration, and the same objective (Nikon $40 \times, \mathrm{NA}=0.6$ ) is used to inject the pump beam and collect the Stokes Raman signal. The latter is sent to a spectrometer (with Andor iDus 401 camera) for analysis. The integration time of each measurement is $0.1 \mathrm{~s}$, and the spectra are averaged over 10 measurements. The sample is placed within a custom-made flow chamber for characterization. Three gases are chosen for this Letter: IPA, ethanol, and acetone. Calibration cylinders containing $1000 \mathrm{ppm}$ of chemical vapors in nitrogen are obtained from Praxair. Test gases below $1000 \mathrm{ppm}$ are obtained by diluting the calibration gases with extra nitrogen. Test gases above $1000 \mathrm{ppm}$ are produced by bubbling nitrogen through liquid analytes dissolved in aqueous solution. In the latter case, the vapor concentration is estimated through Raoult's law. The pressure of nitrogen to the bubbler is $1.1 \mathrm{bar}$, while the pressure of the gas diluted from the calibration cylinder is 2 bar. We note that $1000 \mathrm{ppm}$ of chemical vapor produced in both methods result in the same Raman signal, validating the prediction of Raoult's law.

We first record the Raman signal of the functionalized waveguide itself by sending only nitrogen onto the waveguide. The spectrum is shown as the orange dashed curve in Fig. 2. A broad background due to the SiN waveguide core can be clearly observed, and no extra features can be discerned from the spectrum when compared to waveguides without the sorbent coating [16,24], indicating that a negligible Raman signal is introduced by the coating. Then IPA vapor is introduced into the gas supply at a high concentration of $3808 \mathrm{ppm}$. The Raman spectrum after the introduction of IPA vapor is shown as the solid blue line in Fig. 2. We can readily observe not only the intense signature Raman peak of IPA at $819 \mathrm{~cm}^{-1}$ corresponding to the in-phase $\mathrm{C}-\mathrm{C}-\mathrm{O}$ stretch vibration, but also the Raman modes at 953,1134 , and $1453 \mathrm{~cm}^{-1}$. It is worth pointing out that no Raman signal can be observed from uncoated waveguides with even saturated IPA vapor. This proves that the coating can significantly enrich the concentration of vapor analytes.

The results for IPA, ethanol, and acetone at various concentrations are shown in Fig. 3. In this figure, reference spectra of the analytes measured in liquid phase with the same microscope are shown as the solid black lines above each graph. We can observe a good correspondence in both the peak position and 


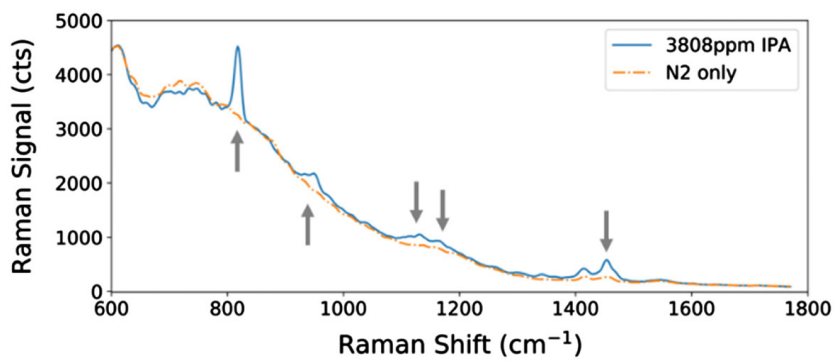

Fig. 2. Raman signal before (orange dashed line) and after (solid blue line) the introduction of IPA vapor. The Raman peaks of IPA (marked with arrows) are clearly visible from the spectrum.
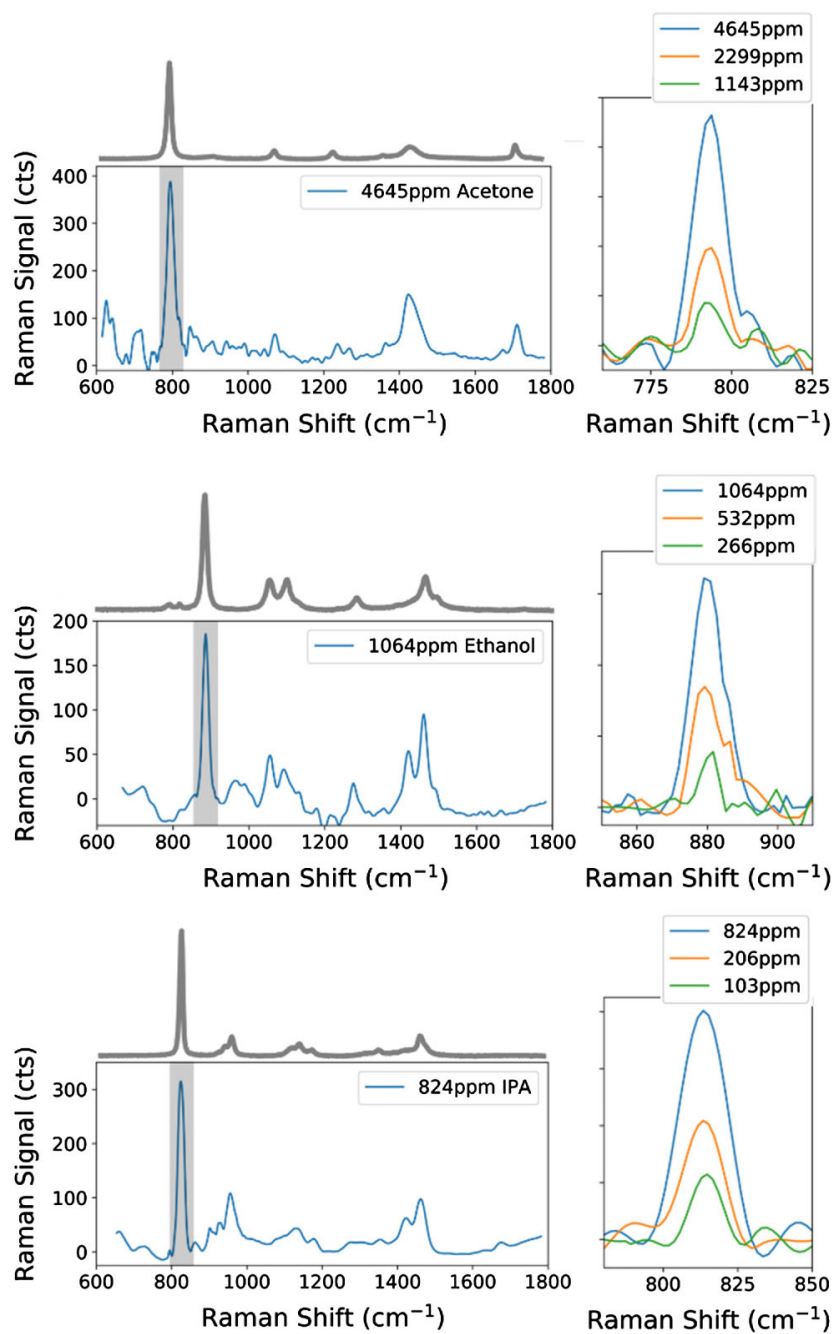

Fig. 3. Background-subtracted Raman spectra of acetone, ethanol, and IPA at various concentrations. The reference spectra of these analytes are shown on top of each graph. The concentration dependencies for the shaded Raman modes are shown to the right of each graph.

relative peak height between background-subtracted spectra and the reference. The result shows that Raman spectroscopy based on a functionalized waveguide is capable of chemical identification.

To characterize the performance of the functionalized waveguide, we apply three different VOC gases with varying concentrations to the waveguide. For each measurement, nitrogen is first applied to the waveguide; then analytes are applied consecutively. The resulting spectra (Fig. 3) are obtained by subtracting the nitrogen-exposed background spectrum from the ones of interest.

We notice the Raman spectra in Fig. 3 exhibit some parasitic features below $1000 \mathrm{~cm}^{-1}$ and a sharp peak at $1415 \mathrm{~cm}^{-1}$, which are the result of inaccurate background subtraction. It is clear from Fig. 2 that the un-subtracted Raman spectra always exhibit some small ripples, which are caused by the collection optics of the microscope. The Raman signal from the waveguide is collected by a multimode fiber. Interference between different optical paths in the multimode fiber induces speckles at the spectrum. The location and intensity of the ripples are not identical across measurements, causing parasitic features in the differential Raman spectrum. We want to emphasize that this effect can be solved by using a single-mode collection fiber. However, it requires sophisticated modification on the microscope to retain the collection efficiency.

The sensitivity of the waveguide is investigated using the signature peaks of analytes which are shaded in Fig. 3. The concentration dependence of these peaks is shown on the righthand side of each graph. It is noteworthy that the variation of the Raman signal is not strictly linear to vapor concentration. Since the Raman signal is proportional to the number of molecules, this suggests that the number of adsorbed molecules is not proportional to the ambient level. Indeed, the adsorption process in mesoporous silica is usually characterized by a Langmuir or Freundlich equation [21]. However, the adsorption capability does exhibit a one-to-one relationship with the ambient level; thus, quantification of VOC is possible once a calibration curve is constructed. Projecting the adsorption capability to lower concentrations using the Freundlich equation, the $3 \sigma$ limit of detection (LoD) of acetone, ethanol and IPA is determined to be 594, 157, and $53 \mathrm{ppm}$ with 1 s integration time and $50 \mathrm{~mW}$ pump power. Here the signal is the relative Raman peak height and spectral noise as the standard deviation of the Raman peak height over repetitive measurements. The $\mathrm{LoD}$ can still be improved by suppressing the interferometric noise down to the level where shot noise becomes dominant.

To demonstrate the multiplex detection capability of the sensor, we perform measurements on samples composed of IPA and acetone vapor mixtures. We first fill the bubbler with diluted acetone solution to generate $2299 \mathrm{ppm}$ acetone vapor. As shown in Fig. 4, only the acetone peak at $791 \mathrm{~cm}^{-1}$ (solid blue) is observed. With a similar method, we obtain the Raman spectrum with solely $854 \mathrm{ppm} \mathrm{IPA} \mathrm{vapor} \mathrm{in} \mathrm{Fig.} 4$ (solid orange), as evidenced by its signature peak at $819 \mathrm{~cm}^{-1}$. Later, we prepare a mixture of IPA/acetone solution in the bubbler to generate a complex gas sample. Its Raman spectrum is shown as the green dots in Fig. 4. We can clearly see the signature peaks of both IPA and acetone. Furthermore, the peak heights are unaffected by the other component. We further increase the concentration of IPA vapor from 854 to $1282 \mathrm{ppm}$ and then to $2204 \mathrm{ppm}$, and the peak height from the other components, acetone, remains the same. This shows that the Raman signal of VOC is unaffected by the presence of other components over a wide concentration range, making this a promising candidate for multiplex detection of VOC.

The temporal profile of adsorption and desorption is also critical for the sensor. A defined sequence of nitrogen, $824 \mathrm{ppm}$ 


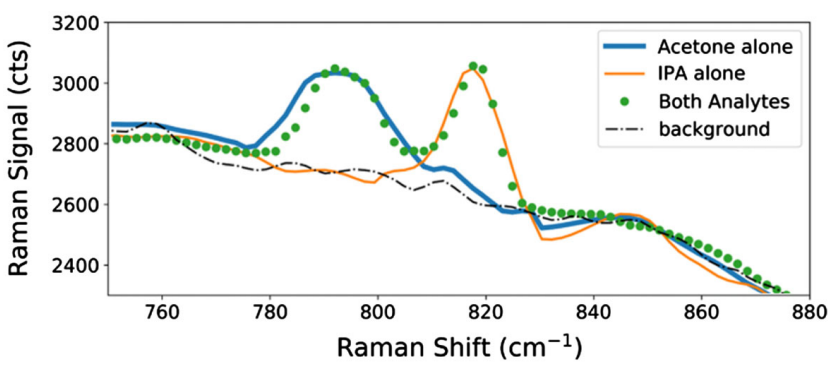

Fig. 4. Raman spectra of gas samples with only acetone vapor (solid blue), only IPA vapor (solid orange), and both vapors (green dot). The waveguide background is not subtracted in the figure. The background of the waveguide with only nitrogen is also plotted (black dashed).

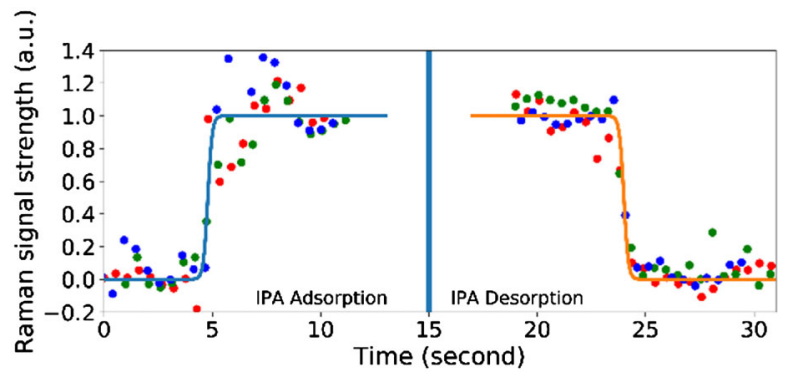

Fig. 5. Temporal profile of adsorption and desorption of $824 \mathrm{ppm}$ IPA vapor onto the mesoporous coating. Three repetitive measurements are shown in the figure with different colors.

IPA in nitrogen, and nitrogen is applied to the waveguide while recording one spectrum per second. The backgroundsubtracted peak height of the $819 \mathrm{~cm}^{-1}$ peak is normalized to its equilibrium value at Fig. 3 , and the temporal evolution is shown in Fig. 5. Both the adsorption and desorption time are less than $1 \mathrm{~s}$. In addition, the Raman peak returns to zero after flushing with nitrogen. This proves that the adsorption is reversible. These characteristics make the sensor promising for long-term VOC sensing. The rapid time response has also been observed for the other two vapors.

The current demonstration has not reached the ultimate performance yet. Optimized waveguide design can increase the modal overlap with analyte and increase the signal. The waveguide is also too short to maximize the signal. To estimate the partition coefficient of the coating, we take the same SiN waveguide without coating and measure the Raman signal from pure liquid IPA. Normalizing the $819 \mathrm{~cm}^{-1}$ mode by the waveguide background to take out the difference in optical alignment, we find that pure liquid IPA, without coating, yields a signal approximately 120 times stronger than $103 \mathrm{ppm}$ IPA vapor. The densities of pure IPA liquid and 103 ppm IPA vapor are 13 and $4.6 \mu \mathrm{mol} / \mathrm{L}$, respectively; considering the difference in mode overlap on uncoated and coated waveguides, the partition coefficient of the coating is estimated to be $2.3 \times 10^{4}$. Modifications of the coating can further improve the partition coefficient [8], enabling sub-10 ppm sensing with 1 sintegration time.

To conclude, the results presented in this Letter show that WERS using functionalized SiN waveguides is promising for multiplex VOC sensing, even in the high humidity condition. Leveraging both waveguide enhancement and chemical adsorption, the detection limits of acetone, ethanol, and IPA vapor are determined to be 594, 157, and $53 \mathrm{ppm}$, respectively, with $1 \mathrm{~s}$ measurement time in the current setup. Optimization in waveguide length, geometry, and the mesoporous silica coating can further improve the detection limit to sub-10 ppm level. Simultaneous quantification of individual components in a complex matrix has also been demonstrated. These results pave the way toward a low-cost on-site all-on-a-chip VOC sensor that may find applications in safety protection and environmental sensing.

Funding. European Research Council (780718, 731465); Fonds Wetenschappelijk Onderzoek.

Disclosures. The authors declare no conflicts of interest.

\section{REFERENCES}

1. D. A. Sarigiannis, S. P. Karakitsios, A. Gotti, I. L. Liakos, and A. Katsoyiannis, Environ. Int. 37, 743 (2011).

2. L. Massolo, M. Rehwagen, A. Porta, A. Ronco, O. Herbarth, and A. Mueller, Environ. Toxicol. 25, 339 (2010).

3. T. Petry, D. Vitale, F. J. Joachim, B. Smith, L. Cruse, R. Mascarenhas, S. Schneider, and M. Singal, Regul. Toxicol. Pharmacol. 69, 55 (2014).

4. World Health Organization, WHO Guidelines for Indoor Air Quality: Selected Pollutants (2010).

5. S. Zimmermann, S. Barth, W. K. M. Baether, and J. Ringer, Anal. Chem. 80, 6671 (2008).

6. Z. Zhang and G. Li, Microchem. J. 95, 127 (2010).

7. F. Alimagham, M. Platkov, J. Prestage, S. Basov, G. Izakson, A. Katzir, S. R. Elliott, and T. Hutter, RSC Adv. 9, 21186 (2019).

8. B. Baumgartner, J. Hayden, and B. Lendl, Sens. Actuators, B 302, 127194 (2019)

9. R. Ferraro, Introductory Raman Spectroscopy (Academic, 2003).

10. R. Salter, J. Chu, and M. Hippler, Analyst 137, 4669 (2012).

11. A. Knebl, D. Yan, J. Popp, and T. Frosch, TrAC Trends Anal. Chem. 103, 230 (2018).

12. G. E. Walrafen and J. Stone, Appl. Spectrosc. 26, 585 (1972).

13. W. Xie, T. Stöferle, G. Rainò, T. Aubert, S. Bisschop, Y. Zhu, R. F. Mahrt, P. Geiregat, E. Brainis, Z. Hens, and D. Van Thourhout, Adv. Mater. 29, 1604866 (2017).

14. X. Nie, E. Ryckeboer, G. Roelkens, and R. Baets, Opt. Express 25, A409 (2017).

15. X. Nie, N. Turk, Y. Li, Z. Liu, and R. Baets, Opt. Lett. 44, 2310 (2019).

16. A. Dhakal, A. Z. Subramanian, P. Wuytens, F. Peyskens, N. Le Thomas, and R. Baets, Opt. Lett. 39, 4025 (2014).

17. A. Dhakal, P. C. Wuytens, F. Peyskens, K. Jans, N. Le Thomas, and R. Baets, ACS Photonics 3, 2141 (2016).

18. S. A. Holmstrom, T. H. Stievater, D. A. Kozak, M. W. Pruessner, N. Tyndall, W. S. Rabinovich, R. A. McGill, and J. B. Khurgin, Optica 3, 891 (2016).

19. N. F. Tyndall, T. H. Stievater, D. A. Kozak, K. Koo, R. A. McGill, M. W. Pruessner, W. S. Rabinovich, and S. A. Holmstrom, Opt. Lett. 43, 4803 (2018).

20. A. Dhakal, A. Raza, F. Peyskens, A. Z. Subramanian, S. Clemmen, N. Le Thomas, and R. Baets, Opt. Express 23, 27391 (2015).

21. B. Baumgartner, J. Hayden, A. Schwaighofer, and B. Lendl, ACS Appl. Nano Mater. 1, 7083 (2018).

22. B. Baumgartner, J. Hayden, J. Loizillon, S. Steinbacher, D. Grosso, and B. Lendl, Langmuir 35, 11986 (2019).

23. D. Stroud, Superlattices Microstruct. 23, 567 (1998).

24. N. Le Thomas, A. Dhakal, A. Raza, F. Peyskens, and R. Baets, Optica 5, $328(2018)$. 